\title{
Interdisciplinary design guidelines of an interface-device for a more accessible urban space.
}

\author{
Ludmila Soñez ${ }^{1}$, Maria Elena Tosello ${ }^{2}$ Esteban San Martin ${ }^{3}$, \\ Agustín Longoni ${ }^{4}$ \\ 1,2,3,4 Universidad Nacional del Litoral \\ Iludmilasonez@hotmail.com \\ 2,3,4 \{maritosello|di.estebansanmartin|agustinlongoni\}@gmail.com
}

\begin{abstract}
This work is part of the interdisciplinary research project "Mediations of augmented space: artifacts, interfaces and representations in artistic and design disciplines", based at the Computing and Design Center of the Facultad de Arquitectura, Diseño y Urbanismo of the Universidad Nacional del Litoral (Santa Fe, Argentina), and directed by PhD. Arch. María Elena Tosello. The work, which was developed in the framework of a grant directed by MSc. Arch. Griselda Bertoni, proposes to reflect on certain limitations that may affect the experience of public space, and how, through the integration of artifacts, interfaces and augmented public spaces, a better appropriation, mobility and habitability of cities can be promoted for seniors. The work seeks to make a creative contribution so that older adults can exercise the right to mobility and feel active citizens of society.
\end{abstract}

Keywords: design-driven innovation, augmented space, elderly, mobility

\section{INTRODUCTION}

In our city, as in many other Latin American cities, public space has a structuring role. It is who configures the areas of pedestrian mobility in the urban environment, defining the quality of the city and, to a large extent, the quality of life of their habitants. In turn, the city is crossed by the phenomenon of virtuality, which is characterized by not requiring the physical presence of users for action, thus affecting the perception that people have of their immediate environment and the experience of public space.

Arroyo (2011) states that the experience of public space today is put into action from the event, from becoming, and not from continuity. The struc- ture of public space must be thought of contingently, connecting elements, among which more the difference should prevail over similarity, producing multiplicity and significant ruptures. Thus, the street is not only the instrument for mobility around the city, but it is the place of manifestation. "The lack of spaces adapted to the requirements... of displacement over short distances (walking, biking, etc.), not only supposes a marginalization of this type of mobility that threatens the very essence of the city, but often entails the impossibility of displacement for broad groups of population" (Herce Vallejo and Magrinyà, 2013:22).

In parallel, the increase in the longevity of the 
population will be, according to the United Nations (UN), one of the most representative social transformations of our century:

- "... the aging of the population is about to become one of the most significant social transformations of the 21st century, with consequences for almost all sectors of society, including the labor and financial market, and the demand for goods and services (housing, transportation, social security), as well as for family structures and intergenerational ties." (UN, 2017)

According to data obtained from the World Health Organization -WHO- [1], it is projected that by the year $2050,22 \%$ of the world population will be people over 60 years old, surpassing for the first time in history the age group that belongs to the children (014 years of age).

Thus, architects and designers in the age of the 4th Industrial Revolution are presented with the challenge of designing and constructing more inclusive interfaces, artifacts, buildings and cities that not only integrate older adults as active participants of society and beneficiaries of its development, but also incorporate the potential of emerging technologies as mediation tools. It becomes a priority, then, that the conditions of accessibility to urban environment allow the use and enjoyment in a safe and autonomous way of any person, regardless of their abilities and circumstances.

Our research aims to propose design guidelines for a system or device (Figure 1) integrated by artifacts, interfaces and accessible public spaces, which benefits the mobility of the elderly, improving globally the quality of urban life. A device is understood as a dynamic network that is established between heterogeneous and interdependent elements, which has a strategic function that responds to an emergency (Foucault, in Agamben, 2007).

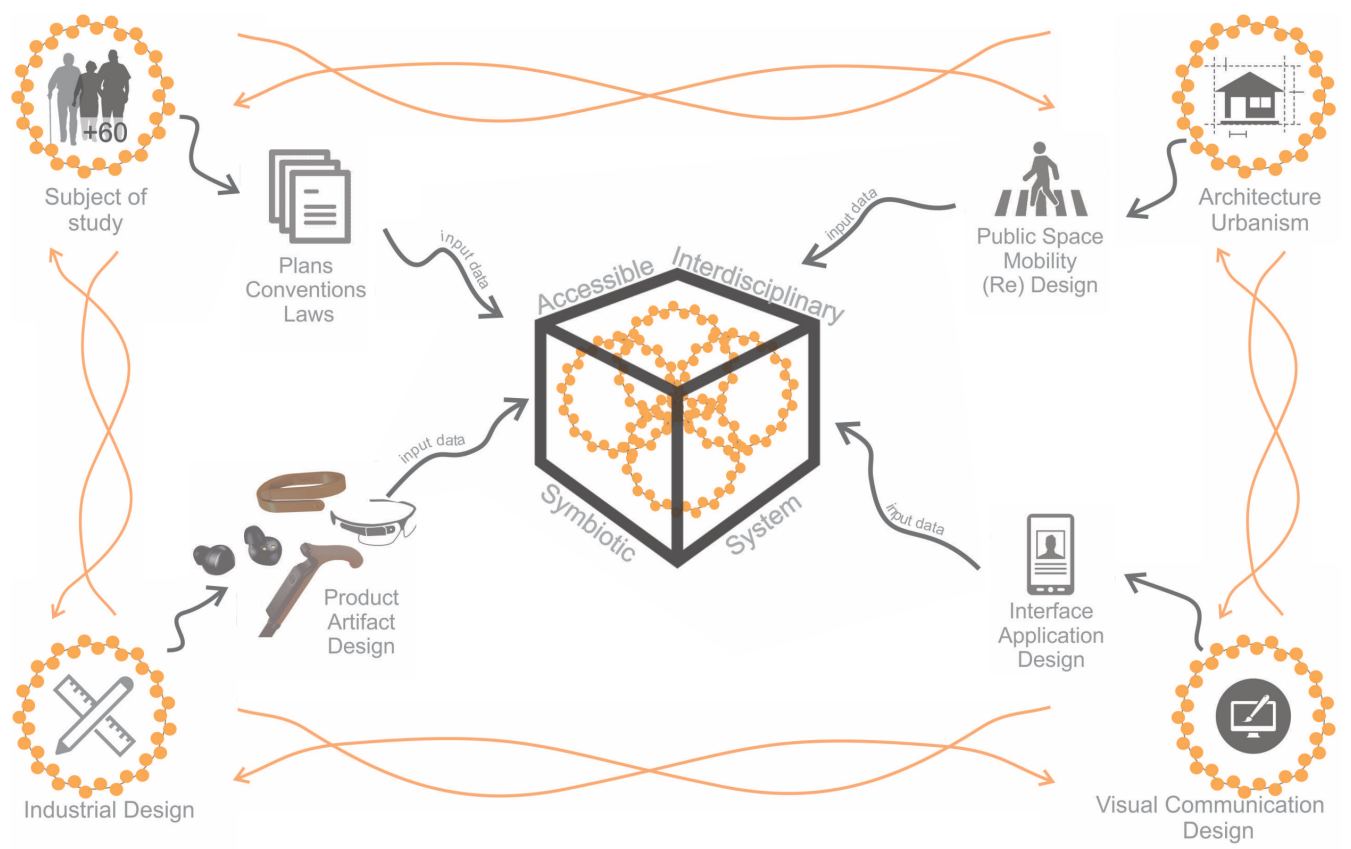

Figure 1

Diagram of the device. 


\section{METHODOLOGY}

The research included different interrelated processes, not necessarily correlative.

Identification of the problem: this stage included the definition of the study problem; the analysis of its effects on subjects and practices; and the interrelation with other problems and contexts. Uses and appropriations of the urban public space were identified based on the incorporation of digital media in social practices; and the profile of the user was outlined, as well as surveys and interviews to record experiences.

Regarding the profile of the user, this is defined as an older adult "in the future", that is, they are people that today are between 40 and 60 years old, which in the next few years will become the new older adults. According to the investigation, the use of the mobile phone and the relationship with the digital media is foreseen to increase on this age group. In addition, it is assumed that the relationship they will have with technology will be different, more assiduous and familiar than the current one.

Background analysis and diagnosis: in this phase the categories of analysis were defined, and legislations, precedents, investigations and experiences with artifacts or facilities powered by digital media that have been integrated into public spaces were analyzed. Their impact and level of appropriation, the materials and technologies used, and their possible applications to facilitate accessibility and mobility in our city (Santa Fe, Argentina) were considered. There were also interviews with specialists, and a diagnosis was made, relating the problems with the available instruments, in view of possible solution alternatives.

New materials, technologies, artifacts (prototypes, projects, etc.), and applications (apps) were investigated, and public spaces in the city with the possibility of being integrated into the device were analyzed. In addition, background examples of smart cities were studied, and information on applications and technologies used to improve the mobility and experience of the city were recorded. It was taken into account that these cities not only made use of
ICT to improve the quality of life of their citizens, but also they were part of the Network of Friendly Cities with Older Adults, belonging to the WHO.

Planning and design: the proposal of accessible (re) design of an urban space was developed and the design premises for the device were established. This device relates artifacts that record information, events, positioning or contextual conditions (a mobile phone + a headset, for example), with a control and management interface that communicates this information to users (application), in order to guide decisions, movements and / or actions in a previously conditioned area of the city.

\section{RESULTS}

The proposal links three disciplinary fields: Architecture and Urbanism -redesign of an urban sector-; the field of Visual Communication Design -definition of the features of the application and characteristics of the interface-; and the field of Industrial Design definition of the functions of the artifacts to be incorporated in the device-.The design premises consist of guiding principles to achieve public spaces that are accessible and free of urban barriers, where pedestrian mobility is prioritized through proposals that promote safety, comfort and autonomy conditions.

\section{Proposal description}

In the field of Architecture and Urban Planning, some principles that according to WHO contribute to facilitate mobility were considered, especially the following recommendations:

- Environments adapted to pedestrians, with resources such as highly visible pedestrian crossings, raised surfaces or pedestrian islands; lower speed limits and measures to moderate traffic; well maintained sidewalks and cords (or curbs); overpasses and accessible underground passes; signs at pedestrian crossings with enough time to cross; and hearing aids in the crossings.

- Aesthetically pleasing elements in streets and parks, such as trees, gardens or vegetation. 
- Numerous street crossings to multiply options, and adequate facilities for the elderly, such as places to rest and public restrooms.

The "groups" proposed in the guide of the Network of Friendly Cities with Adults, presented by WHO, were also considered. In particular, the suggestions in group 1 were observed, those that refer to outdoor spaces and buildings.

- The availability of places to sit down to rest is generally considered as an urban characteristic required for the elderly.

- Friendly sidewalks. The condition of the sidewalks has an obvious impact on the ability to walk in the local area. Narrow, uneven, broken, high bordered, congested or obstructed sidewalks present potential hazards and affect the ability of older people to walk on foot.

- Safe pedestrian crossings.

- Good accessibility. In many cities there are barriers to physical access that can discourage the mobility of older people.

- Adequate public bathrooms. The availability of clean bathrooms, strategically located, correctly signposted, and accessible for people with disabilities.

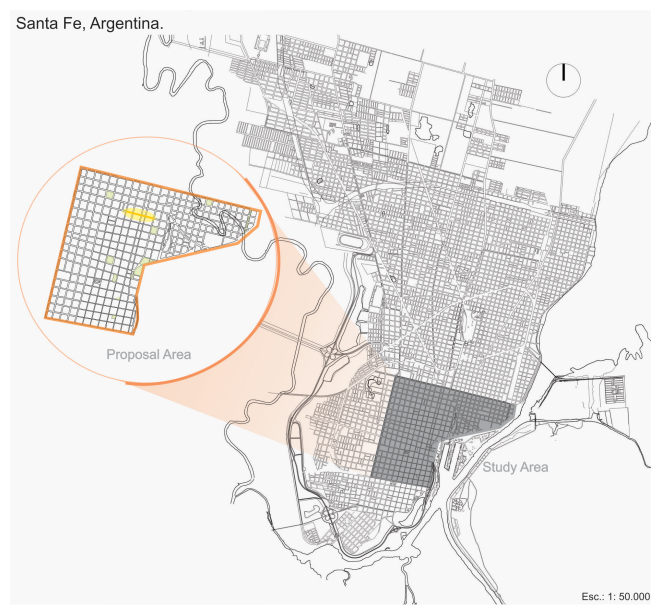

The proposal sought to create an augmented space that merges a good urban design (physical space) with ICT (virtual space), to achieve an intelligent environment.

The urban sector that was going to be intervened was the result of the analysis of a first large area (macro center) delimited by streets and main avenues of the city (Figure 2). Within this sector, a survey was made of the urban services used by the elderly to carry out activities of their daily life (supermarkets, hospitals, clinics, cooperatives, pharmacies, banks, ATMs, etc.). At the same time, the monitoring cameras belonging to the City Government of Santa Fe were also surveyed, which would be useful for the project.

Finally, interviews were conducted with elderly people that are inhabitants of the city of Santa Fe. In the interviews there were two types of questions, some aimed at understanding what the relationship of the older adult is like with cell phones, and other questions related to the mobility in the city, to find out how easy it is for them to travel in the city, how they do it, why, and in what areas of the city they move. The interviews, which were anonymous, were made to 25 adults, all women, who attend various workshops (theater, memory, dance), belonging to the Retired Center "Nicasio Oroño" and the Retired Center "Banco Provincia" of the city of Santa Fe. The gender cut is because the workshops were attended only by women. The interviewees' ages ranged from 67 to 79 years, with an average of 72 years (Figure 3).

Four possible intervention sectors were selected, including one that has a greater number of public and private services, as well as one of the main streets of the city, with significant pedestrian and motorized displacements (cars, most bus lines, motorcycles, etc.).

The redesign of the sector, which incorporates in its premises all WHO recommendations, was made based on two categories that were established to encompass the proposed changes: one on the sidewalk-street relationship, and the other associated with the vehicular movement.
Figure 2

City of Santa Fe, Argentina. Proposal Area. 
Figure 3

Diagrams of some of the information obtained in the interviews.

Figure 4

Schematic cut of the sidewalk-street relationship.

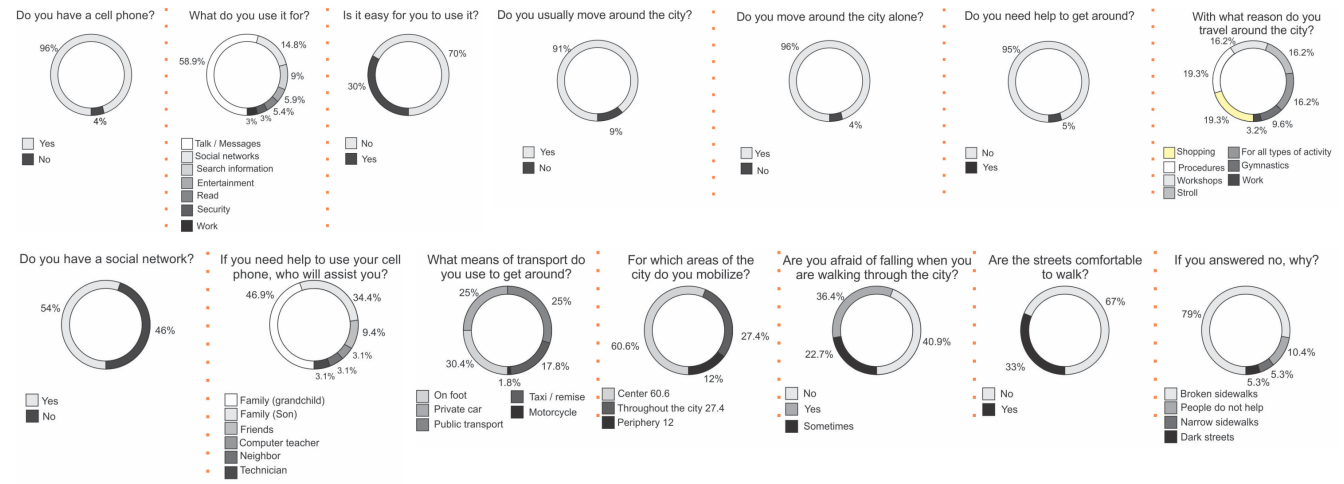

Proposals for sidewalk-street relationship:

- Eliminate the unevenness on the sidewalk, facilitating the safe movement of pedestrians in general, and of the cases studied in this particular research.

- Widen the sidewalks. It is expected to respect the free circulation volume of 2.00 meters wide by 2.20 meters high.

- Relocate trees, signs, gas boxes and traffic lights, to generate the space needed to meet the free volume of circulation.

- Incorporate street furniture, luminaires and public toilets.

- Incorporate both vehicular and pedestrian traffic lights with regressive numerical counter and audible signals for people with sensory disabilities.

- Provide good signage at pedestrian crossings, as well as the signs.

- Place tactile paving to guide people with sensory disabilities.

- Incorporate energy generating tiles in the descent ramps to sustain the system.

- Incorporate protective bollards.

- Place urban garbage dumps (with waste separation).

- Incorporate WiFi signal repeaters or extenders in the structure of existing elements.
Proposals related to vehicular movement (Figure 4):

- Eliminate parking in one of the street's sides, recovering that space for other nonmotorized mobilities.

- Reduce the number of parked cars to promote pedestrian mobility and public transportation, as well as the use of bicycles (bike path projection)

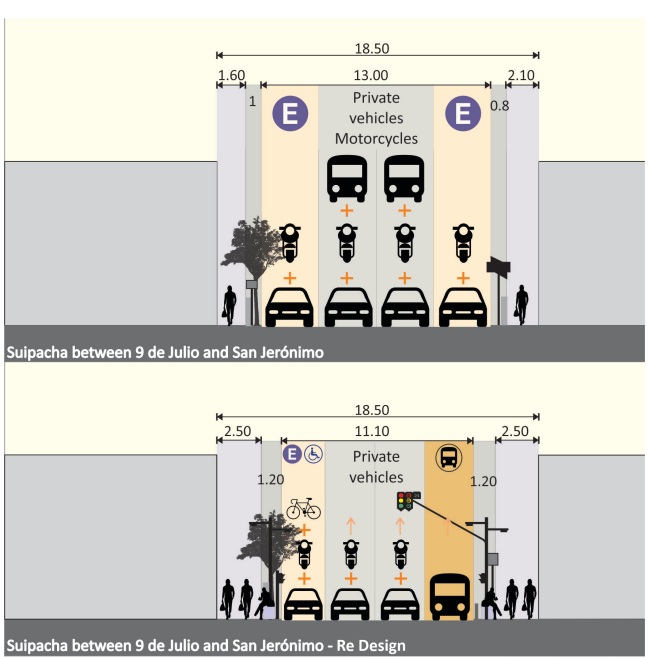



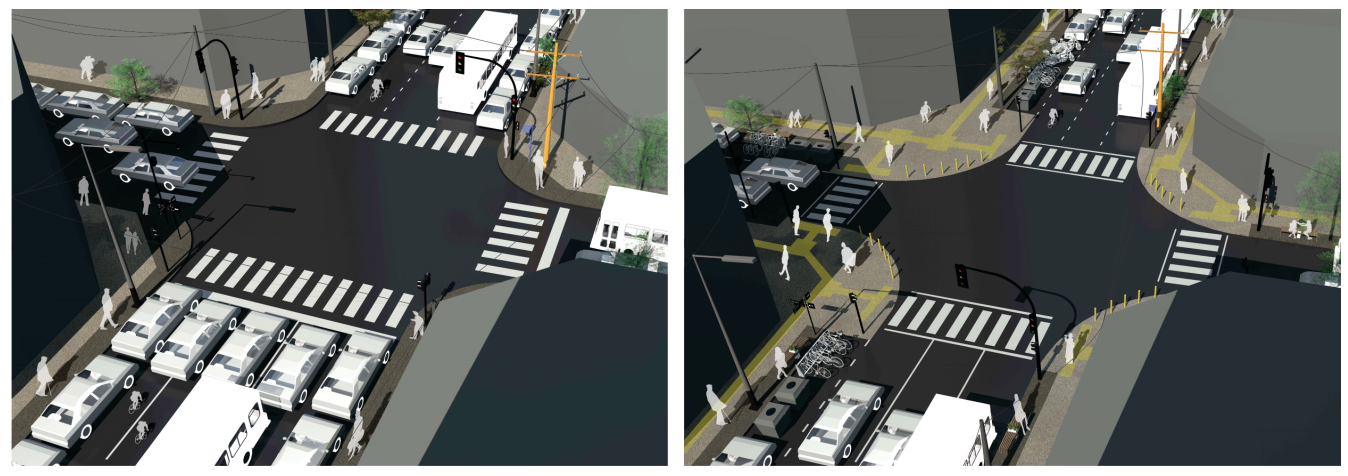

As an example, we present the redesign of one of the streets intersection of the area, since it is replicable both in the remaining intersections of the area, as in other areas of the city (Figure 5).

In the field of Visual Communication Design, the proposal consists of a multiplatform application that, among other features, provides users with ondemand information. "The evolution of information and communication technology (ICT) ...allows to manage and process huge quantities of data... The accessibility to this data opens up various possibilities to extract rich information about cities" (Morello, Carneiro, Desthieux, 2010:499). For this reason, the application will include an interactive map of the city with information about services and equipment that may be of interest to users, as well as information on the status and accessibility of the streets, following the same logic as Google Maps, OpenStreetMap, Maps.me, or other similar applications.

The application (Figure 6) will allow users to configure and edit their profile. In the configuration process they can select custom filters that will be processed by the application to provide information of interest for the user, when the latter is moving through public spaces. As in the scope of this research the subject of study is the elderly, it is assumed that the filters of interest of this type of user will be in relation to their basic needs (accessible routes and services such as supermarkets, pharma- cies, workshops for the seniors, etc.). However, it is expected that the application will not only be aimed at the needs of the elderly, but will be useful to other age groups. Thus, users with different interests could suggest routes, provide circulation advice or share information about activities or events that take place in the city.

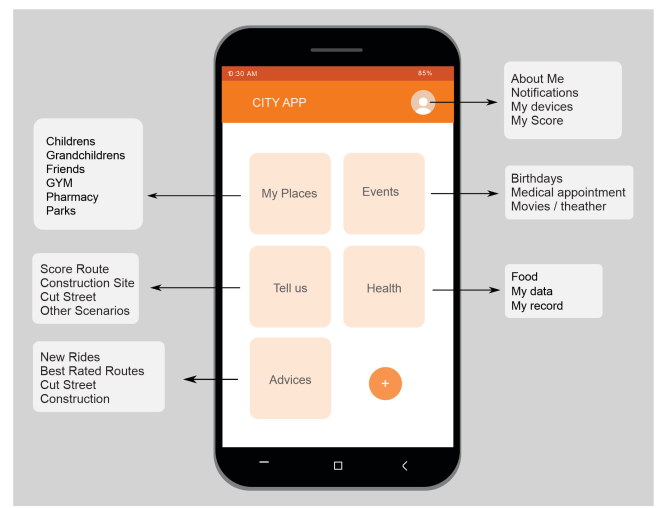

At the same time, the application is intended to link data sources that have been disconnected until now, gathering information that is already on the Web with information shared by registered users based on their own knowledge and experiences. This way, the active participation of users will ensure that the application is completed and updated in real time in a collaborative manner.
Figure 5

Redesign of one of the street intersections.

Figure 6

Possible functions of the application interface. 
As in similar cases, the application will allow the user to have more control over the information, in order to deliberately perform tasks. A large part of the applications that were analyzed seek to adapt to the individual needs of each user. Also, they offer greater security, enabling the user to keep constantly in touch with their relatives or to know their geographic location at all times, similar to Family Locator and Life360. Therefore, in the configuration of the user's profile, two emergency numbers can be registered, enabling the option of sending these numbers to the user's location in real time through Global Positioning System (GPS).

Likewise, it will be possible to define mobility levels with the normal movements of the user and different ranges of information (range of immobility, speed range, range of inclination, range of vital signs, etc.). Also, in the event of any sudden variation in them (either because the user suffered a fall, a robbery or an accident), the application will alert emergency contacts. The alteration in the ranges can not only be warned to the emergency numbers, but the application can be configured to give an alert to other users who are close to the network in order to help the person who has suffered an accident.

Besides of the incorporation of the Web Content Accessibility Guidelines -W3C- [2], other recommendations to consider in the accessible design of web applications for the elderly [3] are the following:

- Information and operation of user interface must be understandable.

- Introduce product features gradually over time.

- Don't divide screens into multiple actions, one focus at once will have better results.

- Give clear feedback on progress and completion of actions.

- Provide reminders and alerts as cues for habitual actions.

- Use breadcrumbs or make it clear how to get back to the home page.

- No text overlaid on images or graphics.

- When using icons and symbols, always pair them with text.

- Simple plain backgrounds are better to avoid distraction.

- Make the purpose of the product clear.

Fonts:

- Allow people to adjust the text size.

- Avoid using multiple fonts.

- Avoid using condensed fonts.

- Use type weight to make a clear hierarchy.

Colors:

- Don't use color to convey a message

- High contrast is better. To verify that the contrast is adequate to guarantee accessibility, use the tool Accessibility Color Wheel [4]

Finally, in relation to the field of Industrial Design, different artifacts were initially investigated and considered to integrate the interface-device. Artifacts like a bracelet or a headset would operate in combination with the mobile phone (the support for the application), but would be used for communication with the user, understanding that both can give an adequate response in the event that the adult person suffers a sensory disability.

However, the first option (bracelet) was finally rejected because, due to vision problems typical of the age group to which the interface-device is intended, the headset is more practical. It is assumed that obtaining information verbally (by voice command), in general, is more useful than visually, since our user the elderly - often suffers functional changes in the eyes that give rise to presbyopia (decreased ability to focus that causes blurred up close vision) or increased lens opacity. Although hearing loss is also common in these users, the headset in turn could function as a hearing aid.

Considering the projects of mobile phones that are in development, which anticipate the possibility of bending the mobile and becoming a wristband, it is expected that in the near future in the interfacedevice the use of another device (like the headset) apart from the mobile phone could be avoided, since 
all the functions would be integrated into the same artifact.

Following the planned functions for the headset, it will connect wirelessly with the application to verbally indicate the user the information available on it. In turn, the user will have the option to use the voice command to change the previously configured data at any time.

All wireless connections necessarily involve the availability of Wi-Fi service, which is why in order for the interface-device to work properly, extenders that amplify the signal could be placed. Beyond the fact that in Argentina the majority of mobile telephony services include data, the extenders would allow improving the signal of the City and / or State public networks, for the benefit of all the inhabitants of the city. These could be located every 200 meters in traffic lights or poles already located in the corners, for example, in the poles that hold the signage that indicates the name of the streets, thus no new elements would be added that could hinder the reading and experience of the urban space.

Based on the analysis of the artifacts that are currently on the market, it is feasible that the design of the handset allows it to be rechargeable with renewable energy by means of small solar panels, and something similar could happen with the extenders. The mobile phone could also be recharged by wireless power transmission. In this way, the entire system would be self-sufficient, not requiring traditional energy for its operation.

As the proposed interface-device emphasizes the information of daily character that the user can receive and not the artifact through which he will receive such information, the background idea is not to produce any new artifact, given that almost all the artifacts that we use on a daily basis allow us to receive information and feedback systems that can help others.

Design-driven innovation questions the reasons why people buy or use a product, and from there seeks to create market opportunities by proposing new meanings for the products (Verganti, 2009). Ac- cording to Verganti "... radical innovations of meaning are rarely pulled by the market, they are pushed by a company's vision about possible breakthrough meanings".

The constant technological evolution allows us to use and resignify the artifacts according to the needs of the users, and thus, each individual adopts the type of artifact that is most useful, usable and desirable. In view of its appropriation, it is important that the interface-device assimilate these three characteristics in order to provide the user with a "brand experience" [5], which in this particular case focuses directly on improving its quality of life (Figure 7).

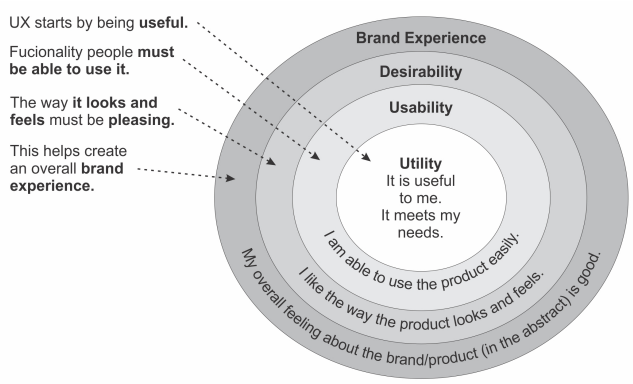

The usefulness refers to whether the product really provides the user what he needs, and the usability responds to how easy and enjoyable is the set of features that the product offers at the time of use. Both are conditioned by the particularities of each user, and in this case, as it has been already mentioned, in the elderly, presbyopia and hearing loss are frequent, as well as the motor problems that cause loss of stability and other conditions that diminish the sensory and motor abilities of these users. In this sense, it is pertinent to remember that the universality of the design is based on its ability to enable products to be used by everyone, regardless of the circumstances and regardless of the platform or the device available to each user (cell phones, headphones bluetooth, glasses, watches and / or smart canes, etc.).

Regarding the desirability of the product, this will depend on the pleasure and well-being that the user feels when using it, and will be based on the
Figure 7

The quality attributes of User Experience (UX) as Utility, Usability, Desirability and Brand Experience [5], with Utility as the core. 
quality of information, the capacity for personalization and the possibility that the user has to mold it according to his own experiences.

Based on the above, we propose a Minimum Viable Product -MVP- [6], a cross-platform network device that has defined the basic characteristics. Also, as it is being used the actions of the users are analyzed so that they shape the evolution of the system, in order to generate an innovation in the design driven by the User Experience -UX-.

Both UX-based design and desirability are linked to the "brand experience" that considers the emotions that a product arouses, since everything already mentioned has its corollary when the user feels and "connects" with the product. Exceeding the expectations of the user is the only way to achieve this purpose. Therefore, the system must go forward step by step to offer more accurate and personalized information, in order to reward the user for using the system, highlighting the value of its information with different contributions to transportation accounts, markets, taxes, etc.

To feed the interface-device (whether with information, energy or others), the City must also consider what is most useful, usable and desirable; for example, it could incorporate paving slabs to convert energy from people's footseps (such as Pavegen System) in the most traveled urban sectors or $5 \mathrm{G}$ networks where antennas or sensors are available. Through Bluetooth beacons that connect to smartphones, piezoelectric tile systems can power off-grid applications - such as lighting, data capture and transmission, and environmental monitoring rewarding citizens for their steps and generating a high commitment to convert their steps into energy and data.

The $5 \mathrm{G}$ networks work with small antennas distributed throughout the city, which, as already anticipated, must be incorporated by strategically choosing their locations so as not to visually contaminate the city. In order to contribute to the energy support of the interface-device, wind turbines can also be incorporated in avenues and spaces with high vehic- ular circulation. These urban turbines use traffic to generate electricity (Enlil Vertical Wind Turbines, for example).

Finally, we consider the loT (Internet of Things) platforms for networks, which are the basis for interconnecting small electronic artifacts that monitor virtually any parameter. Smart cities use them to connect sensors, lights and meters that collect and analyze data, in view of a more effective and efficient use of resources. These data are then processed to improve infrastructure and public services. They can also be used to send emails or SMS messages alerting users when something needs attention [7].

By simplifying communications, these networks cause the low energy consumption device-to-cloud. Soon, there will be no need to replace or recharge batteries, since the artifacts will generate power on their own. Since some networks are compatible with Bluetooth, GPS 2G / 3G / 4G and Wifi, by combining connectivity solutions with each other, the user experience can be drastically improved [8].

\section{CONCLUSIONS}

Unlike the products that are under development and that have more sophisticated and complex technologies, the artifacts that make up the proposed interface-device are everyday objects (headphones, bracelets or canes), which incorporate technologies to provide effective solutions to daily problems. The functions of these objects are easily recognizable and they are controlled in an easy way, which is fundamental for their appropriation by the elderly. Furthermore, their aesthetic design seeks to adapt to a wide spectrum of users (universal design).

As the interface-device emphasizes information and not objects, our intention is not to produce new artifacts but to resignify existing ones, according to the needs of the users. The resignification of technologies, which proposes the creative reuse of knowledge, skills and available artifacts, is a phenomenon that characterizes the processes of technological innovation in Latin America. Innovation can lie in a combination of technologies, that is, in the 
establishment of relationships and interactions between dissimilar elements (Sennett, 2009).

To achieve this purpose, it is relevant to consider the design of the application interface that articulates the relationships between the elements that are combined, since the less complex the procedures are to obtain information, or the less interactions, requirements or steps are necessary to reach the objectives, the better conditions will offer the device to the elderly.

The interface-device consists of a networked system, self-sufficient and constantly connected, where data and information are transmitted as well as energy, to provide security, comfort, guidance and decision-making power to users. Therefore, our proposal would not only benefit the elderly, but could be used in other applications such as wayfinding, for example. Wayfinding studies information communication systems that facilitate the orientation of people to improve understanding and experience of space (Lynch, 1960), and focuses on a non-standardized user profile, considering a diversity of capabilities and physical, cultural, social, etc. variables, in relation to a specific context.

In the dynamics that characterize the current world, information is the actor that gives decisionmaking capacity and autonomy to users. While users are well informed of what happens in their immediate environment and they know its characteristics, the more accurate decisions they can make when they move around the city.

In most of the cases that were analyzed, architecture considers passive solutions regarding the problem of accessibility to the physical environment. However, an accessible design should not be thought only with passive elements, but incorporating instruments of mediation of augmented space. Therefore, we will be creating smart cities in which the promotion of mobility is not only contemplated by a good urban project, but guaranteed by the convergence between the physical, the biological and the virtual.

As future work, we will deepen the study of alter- native energy generation systems, such as the aforementioned piezoelectric tile systems, Enlil wind turbines, or networks like LoRa and Sigfox. Also, we will consider financing lines and possible ways to manage the project for its development.

Finally, we understand that the complexities of our globalized and connected world cannot be solved in isolation. A single professional no longer has the capacity to solve a problem on its own, but rather interdisciplinary groups and collaborative and participatory design processes are needed to address the problems of 21 st century habitat.

\section{ACKNOWLEDGEMENT}

To the Universidad Nacional del Litoral, Santa Fe, Argentina, for the grant of the research project "Mediations of augmented space: artifacts, interfaces and representations in artistic and design disciplines".

\section{REFERENCES}

Agamben, G 2007, ¿Qué es un dispositivo?, Adriana Hidalgo Editora

Arroyo, J 2011, El espacio público. Entre afirmaciones y desplazamientos, Ediciones UNL

Lynch, K 1960, The Image of the City, The MIT Press

Morello, E, Carneiro, C and Desthieux, G 2010 'The Use of Digital 3-D Information to Assess Urban Environmental Quality Indicators', 28th eCAADe Conference

United Nations, UN 2017, World Population Prospects: The 2017 Revision, Key Findings and Advance Tables, United Nations

Sennett, R 2009, El artesano, Anagrama

Herce Vallejo, M and Magrinyà, F 2013, El Espacio de la Movilidad Urbana, Cafe de las ciudades

Verganti, R 2009, Design-Driven Innovation, Harvard Business School Publishing Corporation

[1] https://www.who.int

[2] https://www.w3.org/WHI

[3] https://uxplanet.org/accessible-design-designing-f or-the-elderly-41704a375b5d

[4] http://gmazzocato.altervista.org/colorwheel/wheel. php

[5] https://youtube.com/watch?v=m9wZrQ6DxgY

[6] https://www.ntnu.no/documents/10401/12644339

62/JohanArtikkel.pdf

[7] https://lorawan.es

[8] https://www.sigfox.com/en 